\title{
Contour let-Based Method for Speckle Reduction with Adaptive Estimation of Noise Level
}

\author{
Jassim M. Abdul-Jabbar \\ Computer Engineering Department \\ College of Engineering \\ University of Mosul, Mosul, Iraq. \\ drjssm@gmail.com
}

Amenah. I. Kanaan

Electronics Engineering Department

College of Electronics Engineering

University of Mosul, Mosul, Iraq. amina.edrees@gmail.com

\author{
Zena N. Abdulkader \\ Computer Science Department \\ College of Education \\ University of Mosul, Mosul, Iraq. \\ zenatiq2@yahoo.com
}

\begin{abstract}
Synthetic aperture radar (SAR) and ultrasonic images are inherently affected by speckle noise, which is caused by the coherent nature of the scattering phenomena. This paper presents a contourlet-based method for speckle reduction with an adaptive method for noisethreshold level estimation in a homomorphic framework. The method starts with the generation of many random images simulating the standard deviation level of the logtransformed speckled image. Different contourlet threshold levels are then calculated based on such simulations. Different contourlet coefficients of speckled images are thresholded by their corresponding pre-calculated contourlet thresholds. An exponential operation on the reconstructed output after thresholding is used to simulate the final homomorphic antilogtransformation stage and to obtain the de-speckled images. Unlike other classical and recent de-speckling methods, the despekled images indicate clearly the superiority of the proposed method for speckle reduction, especially for SAR images which possess a lot of detailed textures.
\end{abstract}

Keywords: - Speckled Images, Contourlet Transform, Homomorphic Framework, Adaptive thresholding.

$$
\begin{aligned}
& \text { طريقة لتقليل الضوضاء الرقطية بإعتماد تحويل كونتورلت مع التخمين المتكيف لمستوى } \\
& \text { الضوضاء } \\
& \text { د. جاسم محمد عبد الجبار } \\
& \text { قسم هندسة الحاسوبـ كلية الهندسة } \\
& \text { جامعة الموصل- الموصل- العراق البن } \\
& \text { drjssm@gmail.com }
\end{aligned}
$$

amina.edrees@gmail.com

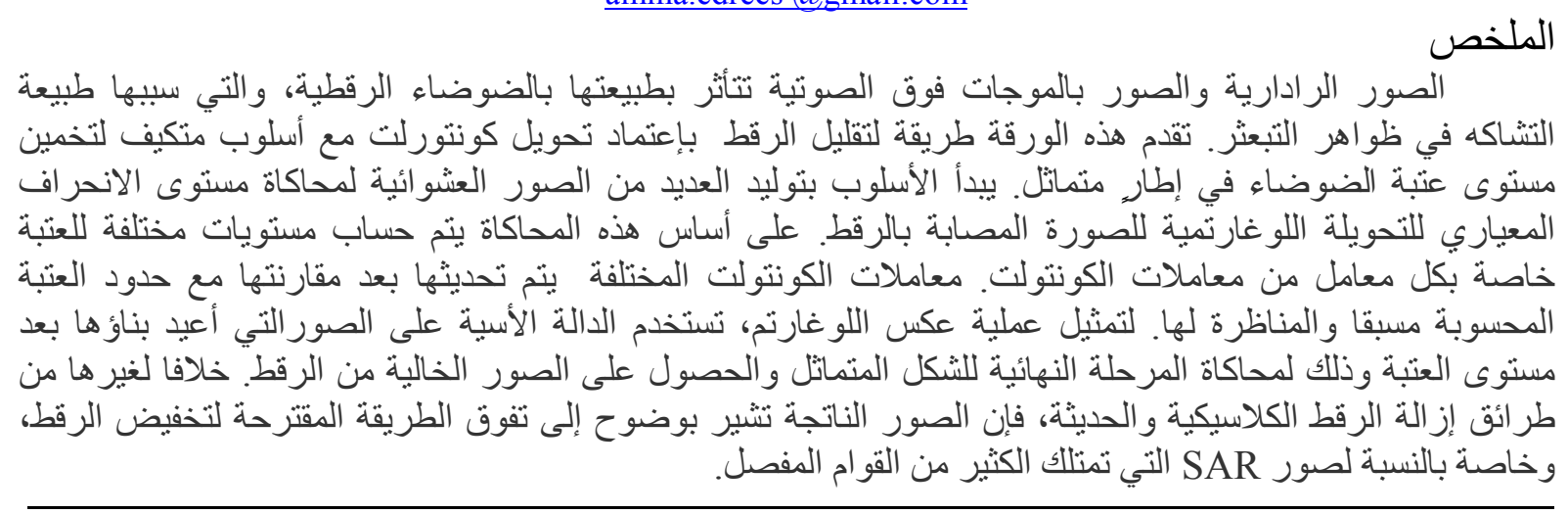

Received: 17 - 7 - 2013

Accepted: 15 - 12 - 2013 


\section{Introduction}

During image formation, transmission and recording processes, images are deteriorated by different kinds of noise. Speckle noise as an important one of those kinds, occurs in all types of imagery acquired from coherent imaging systems, such as laser, ultrasound and synthetic aperture radar (SAR) imagery. It is a consequence of image formation under coherent radiation. It is not truly a noise in the typical engineering sense, since it often carries useful information about the scene being imaged. However, the presence of speckle is generally considered undesirable since it usually damages radiometric resolution, thereby affecting the tasks of human interpretation and scene analysis [1]. Speckle affects all coherent imaging systems and thus regarded as a random pixel-to-pixel multiplicative noise with some a granular appearance that makes the automatic interpretation of the image data be very difficult [2]. Many spatial domain filters were proposed in the past for de-speckling aiming at retaining as much important detailed features as possible. However, their performance is heavily dependent on the choice of the size and orientation of the window [3].

As a more effective two-dimensional tool than wavelet transform, the contourlet transform (CT) is introduced in recent years into the de-speckling applications of SAR and medical images [3]-[5]. CT aims at improving the sparse representation of images over the wavelet transform (WT). Unlike wavelets which can only deal with point singularities exclusively, the main feature of $\mathrm{CT}$ is the potential to efficient handling of two-dimensional (2-D) singularities, i.e., contours. This difference is caused by following two main properties that the CT possess: first; the directionality property that it has basis functions at many directions. On the contrary, the only existing two decompositions of 2-D wavelets are in horizontal and vertical directions. The second is the anisotropy property, which means that depending on the scale, basic functions can appear at various aspect ratios, whereas in wavelets as separable functions, their aspect ratio equals to 1 . Since the CT structure resembles the WT structure, many image processing tasks applied on wavelets can be seamlessly adapted to contourlets [6]. Figure 1 (a) \& (b) shows, respectively the successive refinement by the wavelet and contourlet systems near a smooth contour of an image. This contour is depicted as a thick curve separating two smooth regions.

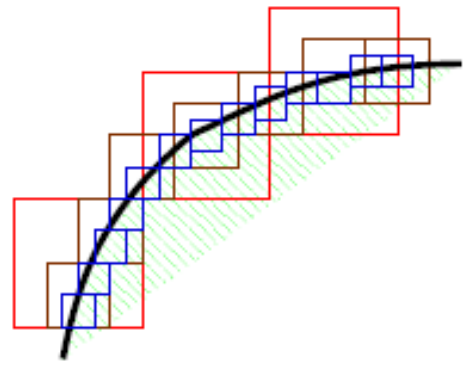

(a) Wavelet

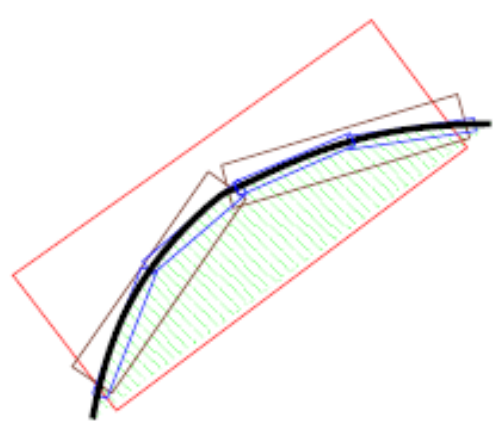

(b) Contourlet

Figure 1 Successive refinement by the wavelet and contourlet systems near a smooth contour of an image.

More recently, there has been much research on many new techniques for speckle reduction in SAR and ultrasound images. In 2010 Can Ding, et al, [7] proposed a method complemented by wavelet transform in conjunction with directional filter banks. First, 
wavelet coefficients of the entry image were derived by wavelet transform. Then the image was only reconstructed by low-pass coefficients and after processing it, high-pass subbands can be derived. In the same year Z. De-Xiang, et al, [8] proposed an efficient SAR image despeckling via Bayesian shrinkage based on nonsubsampled contourlet transform. Nonsubsampled contourlet transform is a flexible multiscale, multidirectional and shiftinvariant image decomposition scheme that can be efficiently implemented via transform. A Bayesian estimator is applied to the decomposed contourlet coefficients of the logarithmically transformed image to estimate the best value for the noise-free signal. In 2011, L. Shahi, et al, [9] used a contourlet method to reduce the noise of echocardiographic images and compared its performance with some other common denoising methods. In 2011 also, P. S. Hiremath, et al, [10] compared the performance of three multiscale methods (wavelet transform, Laplacian pyramid transform and contourlet transform) while de-speckling medical ultrasound images. The effects of different thresholding techniques using Bayes shrinkage rule for denoising ultrasound images were also examined. In 2013, an image denoising technique utilizing random images simulation of the original noisy image is presented [11]. The technique is based on estimating the contourlet thresholding level. The comparison of such technique with three other recent techniques indicates that such technique is more effective in eliminating high-levels of additive white Gaussian noise. Unfortunately, such technique is less effective in reducing speckle noise.

For more speckle reduction and as a modification to the work presented in Ref. [11], a more effective contourlet-based method is proposed in this paper with an adaptive algorithm for estimating noise-threshold level. In addition, a homomorphic framework is also adopted. It is believed that, such framework besides the adaptive estimation of noise level can give much better de-speckling results due to the many non-linear operations involved which fit the multiplicative nature of speckles. In this method, many random images simulating the standard deviation level of the log-transformed speckled image are generated. Different contourlet threshold levels are then adaptively calculated based on such simulations. Different contourlet coefficients of speckled images are then thresholded by their corresponding precalculated contourlet thresholds. An exponential operation on the reconstructed output after thresholding is used to simulate the final homomorphic antilog-transformation stage and to obtain the de-speckled images.

The rest of this paper is organized as follows: In Section II, speckle noise model is described. Section III explains the structure of contourlet transform that is the combination of Laplacian Pyramid and Directional Filter Bank. Section IV introduces the proposed method. Different objective assessment parameters are explained in section $\mathrm{V}$ with the performance evaluation of the method. Section VI contains a comparative study that highlights the superiority of this method. Finally, some conclusions are given in section VII.

\section{Speckle Noise Model}

To explain the mechanism of speckle noise in ultrasound and SAR images, an essential multiplicative/additive compound noise process model is formulized as follows: Let $G(r, c)$, $X(r, c)$ and $N(r, c)$ denote the $(r, c)^{t h}$ pixel of a speckled image, the corresponding original image and the multiplicative noise, respectively. Thus, it can be written that

$$
G(r, c)=X(r, c)+X(r, c) \cdot N(r, c)+\xi(r, c)
$$


where $\xi(r, c)$ is an additive noise (e.g., sensor noise). Usually, the additive term is negligible compared to the multiplicative one. Hence, the additive term is discarded. Thus,

$$
G(r, c)=X(r, c) . S(r, c)
$$

where $S(r, c)$ is the speckle noise that assumed to be fully developed and independent of $X(r$, $c)$ and can be given by

$$
S(r, c)=1+N(r, c)
$$

It should be noted that, a fully developed speckle can be modeled with a Rayleigh distribution. This type of noise can also be simulated by low-pass filtering a complex Gaussian random field, and then using the magnitude of the filtered output. This means that the speckle noise $S(r, c)$ can be approximated as in (3) by a unit-mean Gaussian noise. This will maintain an overall unit-mean for the speckled image [12]. After applying the logtransformation, (2) can be modified to

$$
G_{l}(r, c)=X_{l}(r, c)+S_{l}(r, c)
$$

where $G_{l}(r, c), X_{l}(r, c)$ and $S_{l}(r, c)$ denote the logarithms of $G(r, c), X(r, c)$ and $S(r, c)$, respectively. Since the original detected image pixel values can be factorized into two components as follows:

$$
X(r, c)=L(r, c) \times R(r, c)
$$

where $L(r, c)$ is the luminance and $R(r, c)$ is the reflectance of the scene. Taking the logtransformation of (5) yields

$$
X_{l}(r, c)=L_{l}(r, c)+R_{l}(r, c)
$$

where $L_{l}(r, c)$ and $R_{l}(r, c)$ denote the logarithms of $L(r, c)$ and $R(r, c)$, respectively. Thus, (4) can then be rewritten as

$$
G_{l}(r, c)=L_{l}(r, c)+R_{l}(r, c)+S_{l}(r, c)
$$

which means that each log-transformed pixel in the speckled image $G_{l}(r, c)$ consists three additive components; A low frequency one $L_{l}(r, c)$ and two high frequency components $R_{l}(r$, $c$ ) and $S_{l}(r, c)$. However, applying any simple low-pass filter (LPF) on the log-transformed speckled image pixel $G_{l}(r, c)$ in (7) can linearly isolate the high frequency noise component $S_{l}(r, c)$, but it will also blur many important signal features due to elimination of high frequency image pixel component $R_{l}(r, c)$. An alternative way for low-high frequency isolation of (7) is to use a wavelet thresholding in a homomorphic framework. Many modified and homomorphic wavelet-based de-speckling techniques are previously designed [2], [13], [14]. Most of those techniques suffer from high computational complexity drawbacks. In one of the recent publications, a wavelet-based homomorphic de-speckling technique with a directional weighted-averaging stage is employed to obtain the coefficients of the log-transformed image [15]. In this paper, a new contourlet-based homomorphic method is employed with the adoption of the adaptive estimation of noise level which means an adaptive thresholding technique on different contourlet coefficients is applied.

\section{Contourlet Transform}

The Contourlet transform is a multi-resolution and a multidirectional transform that is powerful for providing sparse expansions of images containing smooth contours. A double 
filter bank structure named pyramid directional filter bank (PDFB) is employed. PDFB is composed of Laplacian Pyramid (LP) that implements the multiresolution decomposition and Directional Filter Banks (DFB) that implements the multi direction decomposition [16]. The contourlet transform proposed initially by M. Do and Vetterli [3] can be considered as a general extension to the 2-D wavelet transform. The contourlet expansion offers a composition of 2-D basis oriented at various directions in multiple scales, with flexible aspect ratios. Due to its rich set of 2-D basis, contourlet transform can effectively capture smooth contours [17]. Figure 2 displays a block diagram of the PDFB structure of the contourlet transform. Band-pass images from the LP are fed into a DFB so that directional information can be captured. For more contourlet coefficients, the scheme can be iterated on the coarse image. The combined result is a double iterated filter bank structure, named contourlet filter bank, which decomposes images into directional sub-bands at multiple scales [6].

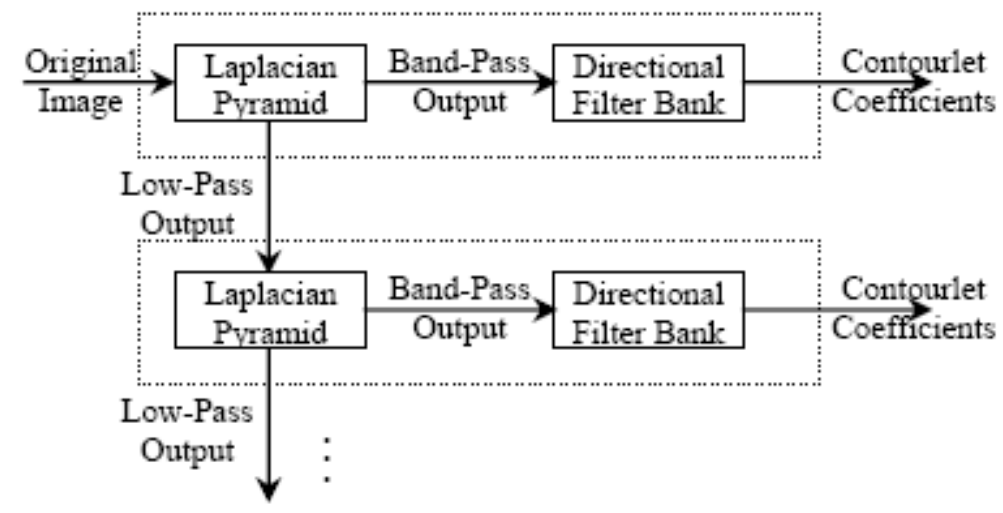

Figure 2 Block diagram of the contourlet transform, after [18]

Similar to wavelet, contour let can decompose the image into different scales. But unlike the wavelet which can only decompose each scale into two directions, contourlet can decompose each scale into any arbitrarily power of two's number of directions. Also, different scales can be decomposed into different numbers of directions [19]. Thus the transform can obtain a sparse image representation by first applying a multi-scale transform and then applying a local directional transform to gather the nearby basis functions at the same scale into linear structures. LP and DFB stages will be discussed in the next subsections.

\section{A. Laplacian Pyramid (LP)}

Burt and Adelson to compress the image after filtration first developed Laplacian Pyramid (LP). It is used to obtain a multi-scale analysis which produces a version of the original image that is sampled down and low-passed. The difference between the original version and the version foreseen is also obtained [9].

The LP can be realized as a multi-scale decomposition filter bank, which produces two output components at each decomposition level. The first represents the low frequency component (low-pass predictions) of the original image. The second image is the difference between the original input image and those predictions. The realization of a one level LP decomposition is illustrated in Figure 3. From such figure, the input image is processed with the two filters $(\mathrm{H}$ and $\mathrm{G})$, which are the analysis and synthesis filters respectively. The LP decomposition produces two output images $\left(a_{1}\right.$ and $\left.a_{2}\right)$, where $a_{1}$ is the coarse approximation 
and $\mathrm{a}_{2}$ is the difference between the original image and the predictions. Also from the figure, the LP avoids the scrambled frequencies by only down sampling the output of the low-pass filter. The generated sub-bands from multi-scale decomposition stage (LP) are followed by a DFB to reveal the directional details (see Figure 2). This process is iterated to produce the next decomposition level [16].

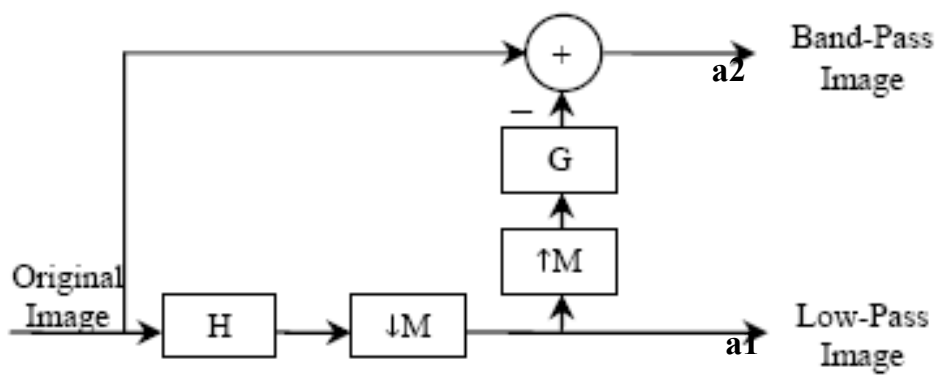

Figure 3 One level of Laplacian Pyramid decomposition, after [9].

The LP has the advantage over the wavelet scheme that each pyramid level generates only one band-pass signal, even for multidimensional cases. This property makes it easy to apply many multidimensional algorithms using a coarse-to-fine strategy to LP. Furthermore, the resulting band-pass signals of the LP do not suffer from the "scrambled" frequencies [7].

\section{B.Directional filter bank (DFB)}

Barberger and Smith developed a two-dimensional filter bank, which can accomplish maximum denoising with an optimum reconstruction. It is known that, the high frequencies of an image, contains some information about directions. In fact, a directional filter bank (DFB) has the required ability to receive the directional high frequencies of the input image. The DFB operates efficiently by analyzing a l-level binary tree, which can produce $2^{l}$ subbands. The frequency partition map for the eight bands directional filter bank and positions of the decomposed sub-band images in the analysis bank are shown in Figure 4. For example, if we have three levels of analysis, then $2^{3}=8$ sub-bands from 1 to 8 will be formed [9].

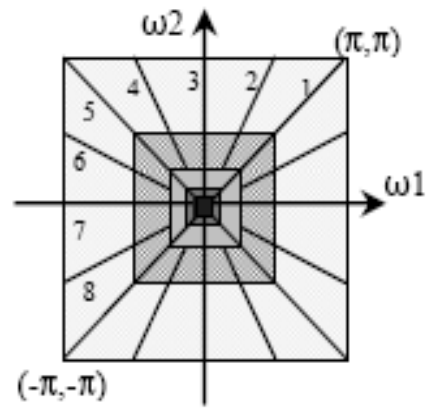

\begin{tabular}{|l|l|l|l|}
\hline \multicolumn{2}{|c|}{1} & \multicolumn{2}{c|}{2} \\
\hline \multicolumn{2}{|c|}{3} & \multicolumn{2}{c|}{4} \\
\hline 5 & 6 & 7 & 8 \\
\hline
\end{tabular}

Figure 4. Frequency portioning map for eight-band directional filter bank, with $l=3$, there are $2^{3}=8$ real wedged-shaped frequency bands. Sub-bands 1-4 correspond to the mostly horizontal directions, while sub-bands 5-8 correspond to the mostly vertical directions. 


\section{The Proposed Method}

Basically, speckle noise filtering (de-speckling ) can be viewed as replacing each from image pixel value in the speckled image with a new value depending on the local context. Such filtering process should vary from pixel to pixel based also on the local context [20]. This goal can be achieved either in the image domain (spatial domain processing) or in the frequency domain (transform domain processing). The latter is used in this proposal. The proposed de-speckling method presents a contourlet-based method with an adaptive thresholding process in a homomorphic environment. A signal flow-diagram of the proposed homomorphic de-speckling method is shown in Figure 5. The speckled image pixels $G(r, c)$ is initially log-transformed to $G_{l}(r, c)$ which is an image with additive noise as given in (7). Other adaptive nonlinear processing stages of Figure 5 are described as follows: As shown in Figure 5, the log-transformed image $G_{l}(r, c)$ is fed to a contourlet transform. Bio 9/7 filters are used in pyramidal decomposition, representing the LP and in directional decomposition, simulating the DFB, respectively. The output contourlet coefficients are further subjected to an adaptive thresholding for another nonlinear transform domain processing in addition to the initial log-transformation. The same filters types are used for reconstruction.

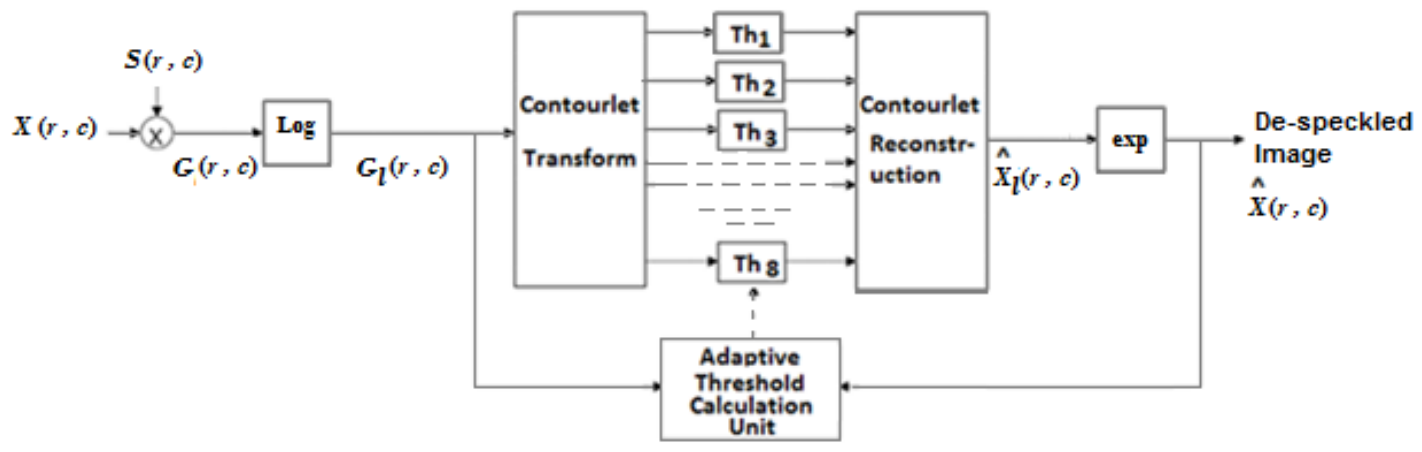

Figure 5 A signal flow-diagram of the proposed homomorphic de-speckling method.

An adaptive threshold calculation unit is designed as a modified version to that introduced in Ref. [11]. Figure 6 shows the main flow-chart of the adaptive threshold calculation unit inside the homomorphic framework. In such unit, the log-transformed noisy image $G_{l}(r, c)$ is read first, where its noise is of the additive type (after log-transformation). At the same time, a scaled version of the standard deviation $\left(\sigma_{n}\right)$ of the log-transformed noisy image is computed. Another contourlet transformation is also applied in this unit. The number of directional filter bank decomposition levels ( $n$ leaves, or nlevs) is then selected. That means a fixed vector of number of directional filter bank decomposition levels at each pyramidal level (from coarse to fine scale) is created. The output of contourlet decomposition inside the unit will contain a cell vector of length $=[$ length of $($ nlevs $)+1]$. Each cell corresponds to one pyramidal level in a cell vector that contains band-pass directional subbands from the DFB at that level. As a result of above analysis, the contourlet coefficients will be obtained. These coefficients are converted to a vector form using the following matlab 2-D to 1-D transformation:

$$
[\mathrm{A}]=\mathrm{Y}(:)
$$


Where $Y$ is an output coefficient vector after both LP and DFB stages. The instruction in (8) converts the contourlet coefficients into a vector form and then stores the results of conversion into a matrix denoted by [A], where A represents a 1-D vector array that contains all coefficients after LP and DFB stages. In addition, the noise standard deviation is estimated by a random generation of images using the same contourlet coefficients that is applied on the log-transformed noisy image $G_{l}(r, c)$ in different levels. A first random image is generated with the same size of the read log-transformed noisy image. Its contourlet coefficients are then obtained and converted to a vector like $\mathrm{A}_{l}$. This operation is repeated for many other random images to find the whole $\mathrm{A}_{k}$ matrices where $k$ is the number of the whole experimented random images. The average noise standard deviation $\left(\sigma_{\mathrm{r}}\right)$ is calculated for all those random images. Such $\left(\sigma_{\mathrm{r}}\right)$ is then stored in another matrix that will be used for evaluating the matrix containing the contourlet thresholds $\left(\mathrm{C}_{\mathrm{Th}}\right)$, where

$$
\mathrm{C}_{\mathrm{Th}}=\sigma_{\mathrm{n}} * \sigma_{\mathrm{r}}
$$

where $*$ dentes a scalar to matrix multiplication operation.

Experiments in Ref. [11] indicated that only ten random images are enough to serve for such purpose. The final contourlet threshold $\mathrm{C}_{\mathrm{Th}}$ is computed by equating the average standard deviation level of those ten random images with the standard deviation of the logtransformed noisy image $G_{l}(r, c)$. The resulting $\mathrm{C}_{\mathrm{Th}}$ levels are then compared on element by element basis with all elements of $\mathrm{A}_{j}$ matrix, $j=1,2,3, \ldots . . k$, as given by the following equation:

$$
\mathrm{A}_{i j}=\left\{\begin{array}{rc}
\mathrm{A}_{i j} & \text { for } \mathrm{A}_{i j}>\mathrm{C}_{\mathrm{Th}} \\
0 & \text { otherwise }
\end{array}\right.
$$

Where $\mathrm{A}_{i j}$ is an element in the matrix $\mathrm{A}_{j}$. This scanning represents an adaptive thresholding method. The resulting vector after thresholding is then converted to contourlet coefficients to obtain a reconstructed log-transformed image $\hat{X}_{l}(r, c)$. As a final stage, an exponential operation on the reconstructed output of the contourlet reconstruction unit is used to simulate the final homomorphic antilog-transformation stage and to obtain the de-speckled image $\hat{X}(r$, $c)$. Since the mean of the log-transformed speckle noise is usually biased [2], an adjustment is needed to remove the associated mean bias to avoid extra distortion in the restored image, especially for high noise levels. This adjustment is provided in the final stage.

\section{Objective Assessment Parameters and Performance Evaluation}

Different objective assessment parameters are used to evaluate the performance of the proposed de-speckling technique. These parameters are Noise Mean Value (NMV), Noise Variance (NV), Noise Standard Deviation (NSD), Mean Square Difference (MSD), Equivalent Number of Looks (ENL), and Deflection Ratio (DR) [2], [14], [21].

NV determines the contents of speckles in an image. A lower NV gives a "smoother and cleaner" image as more speckles are removed, although, it not necessarily depends on the intensity. The formulas for calculating NMV, NV and NSD are given by [15] 


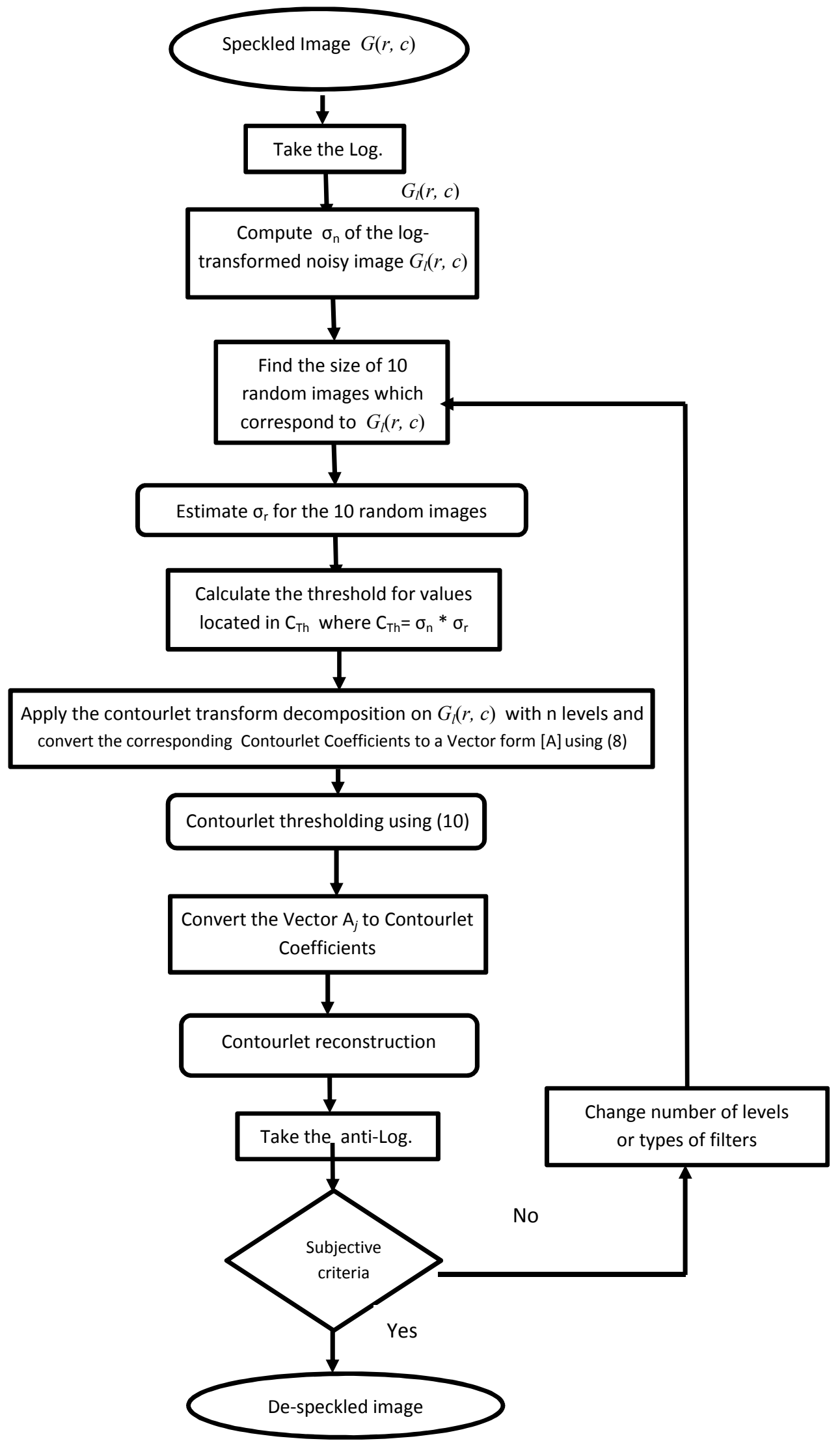

Figure 6 Flow-chart of the adaptive threshold calculation unit inside the homomorphic framework. 


$$
\begin{aligned}
& \mathrm{NMV}=\frac{\sum r, c \hat{X}(r, c)}{R \times C} \\
& \mathrm{NV}=\frac{\sum r, c[\hat{X}(r, c)-N M V]^{2}}{R \times C},
\end{aligned}
$$

and

$$
\mathrm{NSD}=\sqrt{\mathrm{NV}}
$$

where $\hat{X}(r, c)$ represents the de-speckled image pixels and $R \times C$ is image size.

On the other hand, MSD indicates average difference of the pixels throughout the image. It can be calculated as

$$
\mathrm{MSD}=\frac{\sum r, c[X(r, c)-\hat{X}(r, c)]^{2}}{R \times C}
$$

A lower MSD indicates a smaller difference between the original and de-speckled images. This means that there is a significant filter performance. Nevertheless, it is necessary to be very careful with the edges [14]. ENL is another good approach of estimating the speckle noise level in an image over uniform regions. A simple formula for ENL is given by

$$
\mathrm{ENL}=\frac{\mathrm{NMV}^{2}}{\mathrm{NSD}^{2}}
$$

Larger values of ENL usually correspond to a better quantitative performance. The values of ENL also depend on the size of the tested region [4]. DR is also used as a performance estimator. [2] give the formula for DR

$$
\mathrm{DR}=\frac{1}{R * C} \sum r, c\left(\frac{\hat{X}(r, c)-N M V}{N S D}\right)
$$

The ratio DR should be higher at pixels with stronger reflector points and lower elsewhere, thus DR can indicate edge preservation performances of different speckle reduction schemes. 


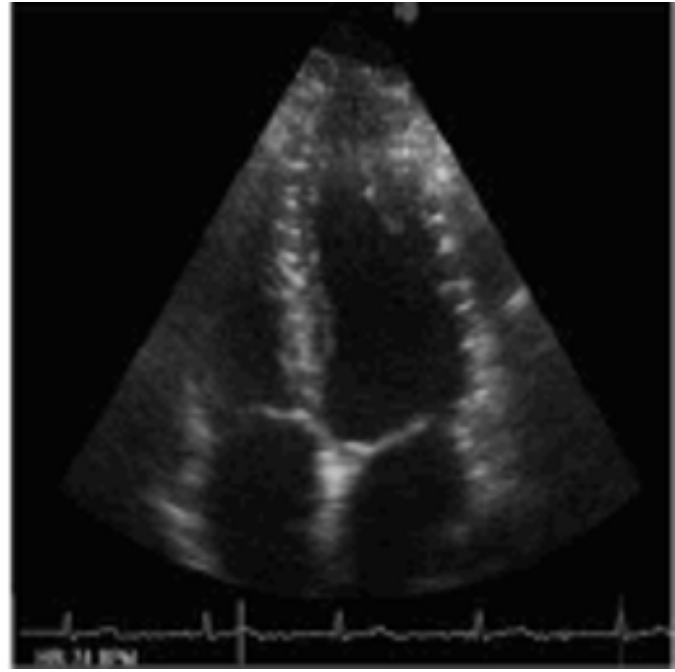

(a)

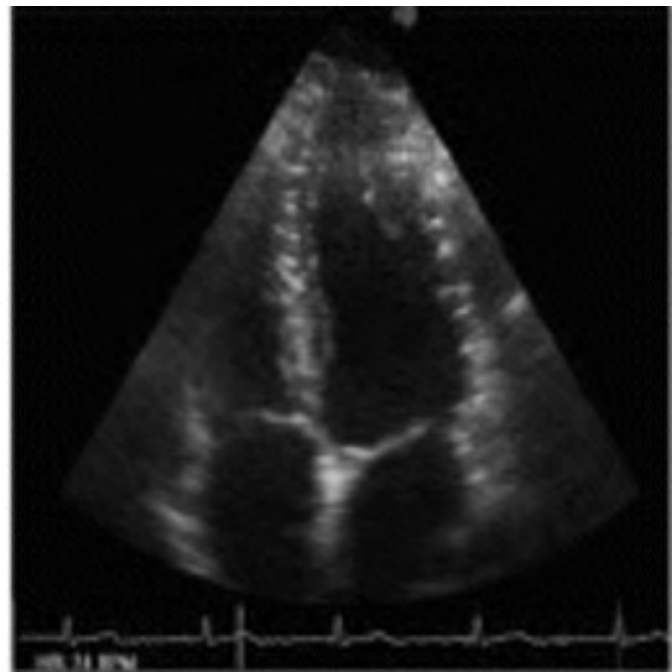

(b)

Figure 7 Echocardiographic image of the human heart.

(a) Original image. (b) De-speckled image.

The performance of the proposed method is evaluated by calculating the pre-mentioned objective assessment parameters for the de-speckled ultrasound image of Figure 7 which shows an echocardiographic image of the human heart, in four-chamber view. This image was originally acquired related to healthy individuals from Shahid Rajayi Hospital [9]. For such image, the proposed method returns the following values for different assessment parameters: $\mathrm{NMV}=24.6436, \mathrm{NSD}=7.7335, \mathrm{MSD}=59.8076, \mathrm{ENL}=10.1543, \mathrm{DR}=$ $1.4875 \mathrm{e}-14$. It should be noted that in addition to the acceptable outlook of the de-speckled image in Figure 7, these parameters also indicate the effectiveness of the proposed method as will be proved in the next section.

\section{A Comparative Study}

The effectiveness of the proposed technique is demonstrated by comparing its objective and subjective performances with four classical de-speckling techniques (such as VisuShrink (Hard-Thresholding), BayesShrink, NormalShrink, and SUREShrink [21]).The comparison also includes three recent techniques; SmoothShrink [2], POSAShrink [14], and Wavelet-Based Homomorphic De-Speckling (WBHDS) technique [15]. ERS SAR Precision Image (PRI) standard of Buenos Aires area is used as an original noisy image (see Figure 8). Such image is from remote sensing satellite ERS-2, with 8-bit, $242 \times 242$ pixels. The despeckled images, processed by using the pre-mentioned classical and recent techniques, including the proposed method are also shown in Figure 8. From the resulting de-speckled images in Figure 8, it can be seen that the proposed method is a successful tool for eliminating speckle without distorting useful image information, i.e., preserving important image edges.

Table 1 shows the objective assessment parameters for different images shown in Figure 8. The quantitative results of Table 1 again highlight the superiority of the proposed method in eliminating speckle with the preservation of useful image information, since it has a good NMV preservation and gives the best variance reduction $(\mathrm{NSD}=6.9144)$. Since a successful de-speckling technique will not significantly affect the mean intensity within a homogeneous 
region, the proposed one acts well in that sense too. As illustrated in Table 1, the proposed method also outperforms the others in terms of ENL. Larger ENL value usually corresponds to a better quantitative performance. From edge preservation point of view, DR value indicates again the superiority of the proposed method.

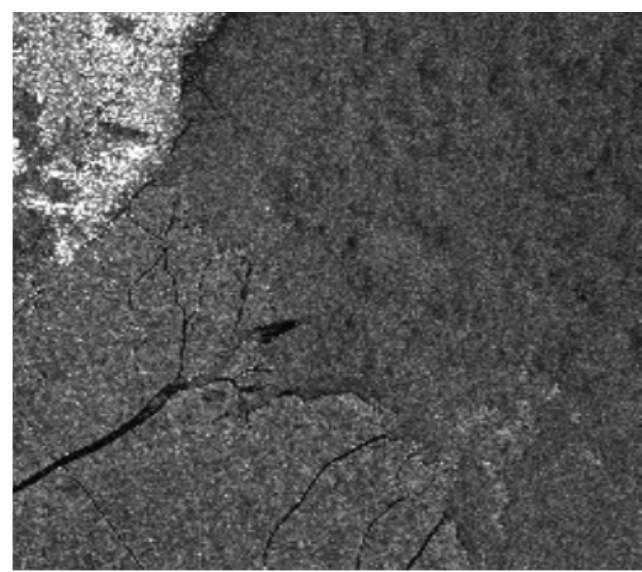

(a) Original

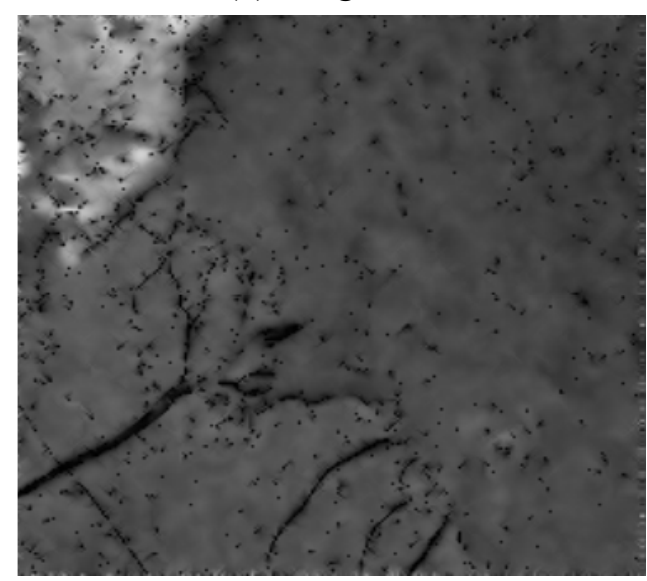

(c) NormalShrink

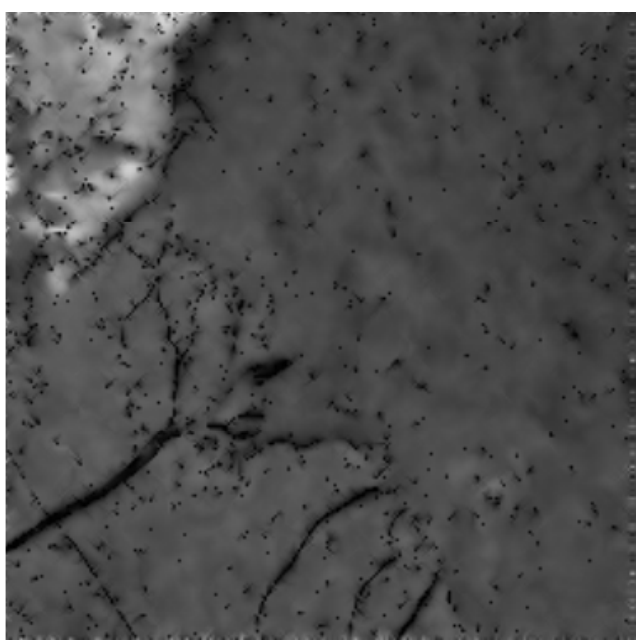

(e) SUREShrink

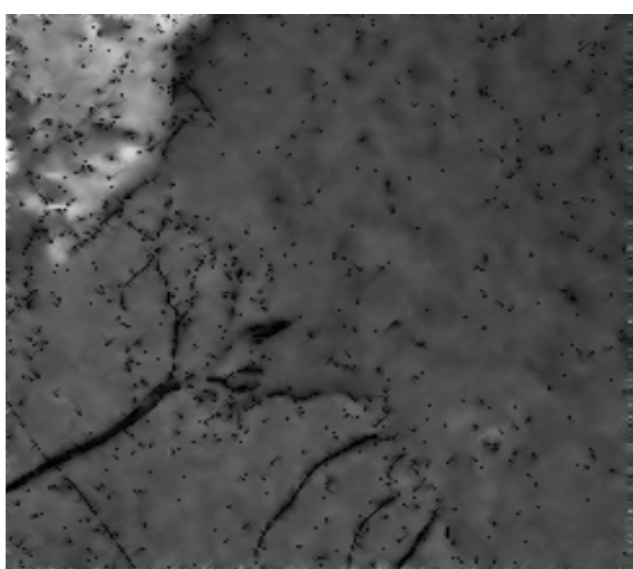

(b) VisuShrink

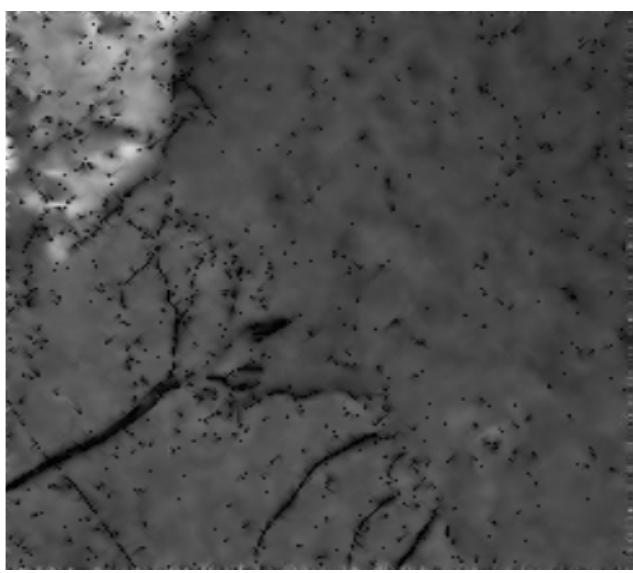

(d) BayesShrink

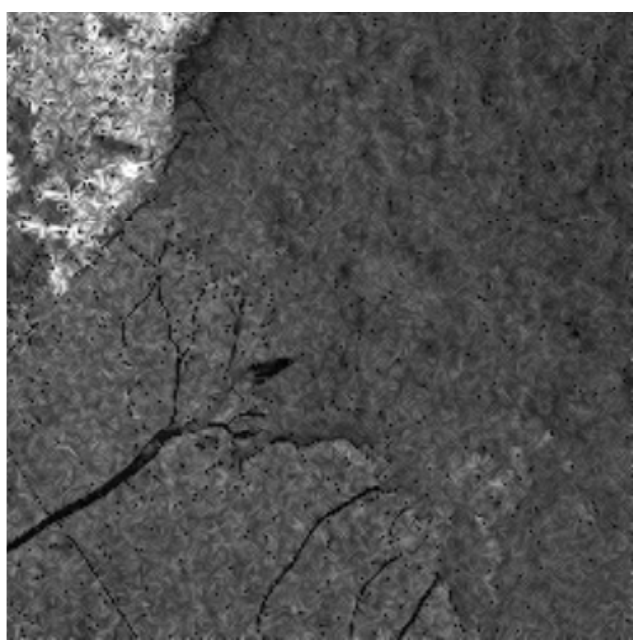

(f) SmoothShrink

Figure 8 Original SAR speckled image and restored images of different techniques. 


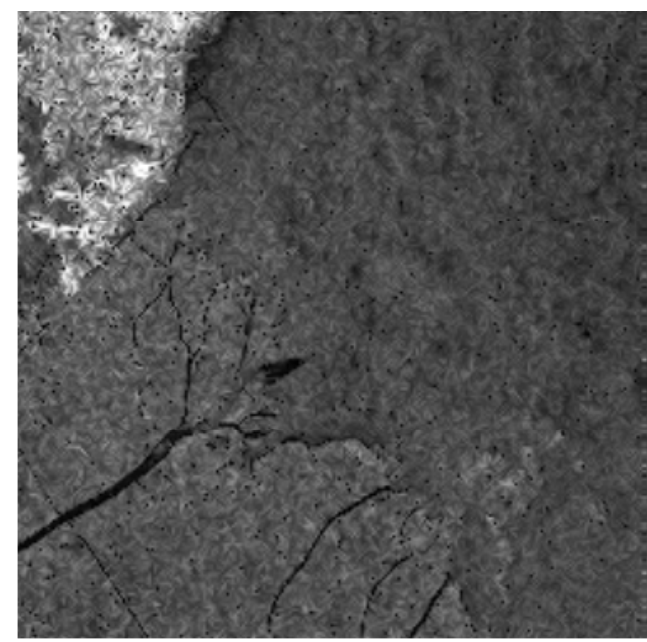

(g) POSAShrink

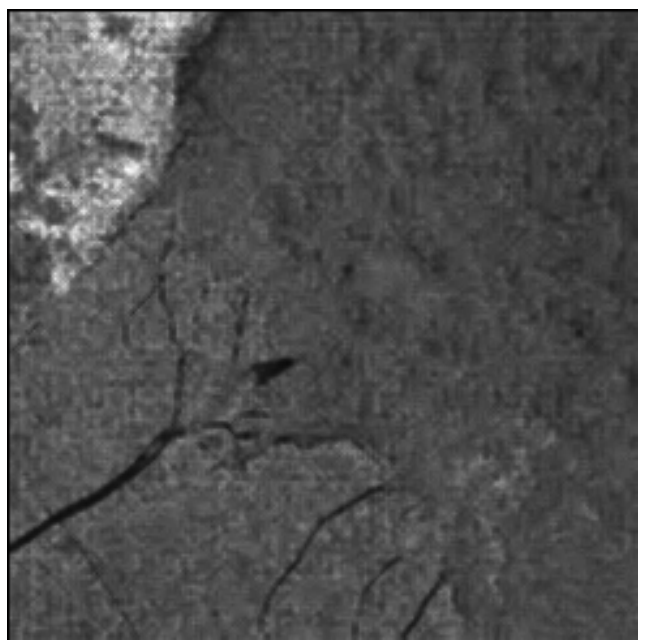

(h) WBHDS technique

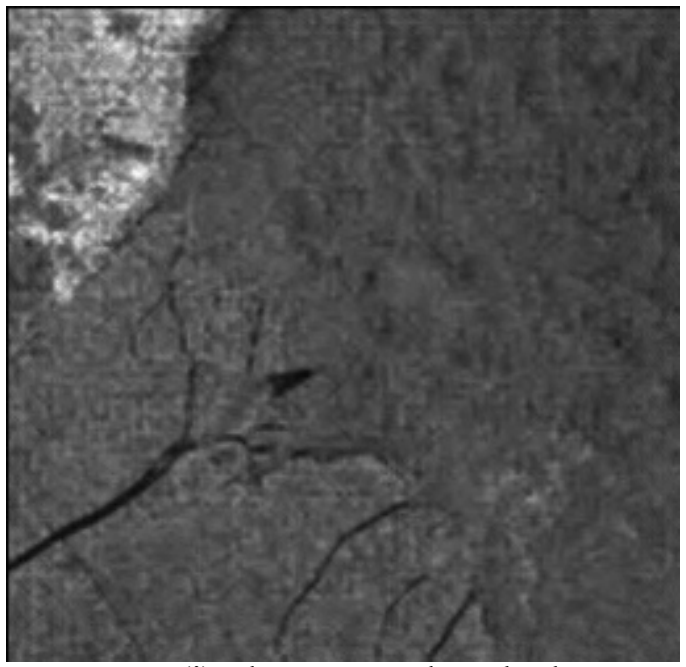

(i) The Proposed method

Figure 8 (continued).

Table 1 Objective assessment parameters for different de-speckling techniques.

\begin{tabular}{|c|c|c|c|c|c|}
\hline \multirow{2}{*}{ Techniques } & \multicolumn{5}{|c|}{ Objective assessment parameters } \\
\cline { 2 - 6 } & MSD & NMV & NSD & ENL & DR \\
\hline $\begin{array}{c}\text { Original noisy } \\
\text { image }\end{array}$ & - & 90.0890 & 43.9961 & 11.0934 & $2.5580 \times 10^{-17}$ \\
\hline VisuShrink (HT) & 855.3030 & 88.4311 & 32.8688 & 39.0884 & $7.8610 \times 10^{-16}$ \\
\hline NormalShrink & 732.2345 & 88.5233 & 33.3124 & 36.8464 & $6.7354 \times 10^{-16}$ \\
\hline BayesShrink & 724.0867 & 88.9992 & 36.8230 & 36.0987 & $1.0534 \times 10^{-15}$ \\
\hline SureShrink & 716.6344 & 87.9920 & 32.8978 & 38.3025 & $2.4005 \times 10^{-15}$ \\
\hline SmoothShrink & 867.1277 & 90.0890 & 32.6884 & 39.0884 & $3.2675 \times 10^{-15}$ \\
\hline POSAShrink & 867.1277 & 90.0890 & 32.6884 & 39.0884 & $3.2675 \times 10^{-15}$ \\
\hline WBHDS technique & 773.2842 & 88.8417 & 26.2757 & 45.7284 & $3.6636 \times 10^{-15}$ \\
\hline Proposed method & 47.8091 & 77.8857 & 6.9144 & 126.8837 & $1.0633 \times 10^{-14}$ \\
\hline
\end{tabular}




\section{Conclusions}

In this paper, a contourlet-based method for speckle reduction with an adaptive method for noise-threshold level estimation in a homomorphic framework for ultrasound and SAR images has been proposed. As well known, the homomorphic environment converts multiplicative speckle model into an additive noise one. In such environment, the proposed method has achieved the thresholded contourlet coefficients in an adaptive manner using the adaptive threshold calculation unit. The resulting contourlet coefficients have been used to reconstruct the log-transformed version of the de-speckled image. As a final homomorphic stage, antilog-transformation is used to obtain the de-speckled image. This method has been tested and compared with some other classical and recent techniques. Subjectively, a cleaner de-speckled image has been obtained which indicates the potential improvement in the detection performance of the proposed method. This refers to the excellent image visual quality of the proposed method because it significantly reduces speckle while preserving the resolution and the structure of the original images.

As illustrated in Table 1, the objective assessment parameters of such method highlight its superiority. Good NMV preservation and best variance reduction (lowest NSD value) show the ability to eliminate speckle with the preservation of the useful image information. A better quantitative performance is also expected as ENL value is the highest. The considerable increase in DR ratio strongly indicates the improvement in performance of the proposed de-speckling method.

\section{Acknowledgement}

The authors would like to thank Mr. Akram A. Dawood for his valuable discussions that helped in the completion of this paper.

\section{References}

[1] A. Achim, E. E. Kuruo glu and J. Zerubia, "SAR Image Filtering Based on the HeavyTailed Rayleigh Model", IEEE Transactions on Image Processing, Vol. 15, No. 9, Sept. 2006, pp. $2686-2693$.

[2] M. Mastriani, A. E. Giraldez, "Smoothing of coefficients in wavelet domain for speckle reduction in Synthetic Aperture Radar images", The International Congress for Global Science and Technology (ICGST), International Journal on Graphics, Vision and Image Processing (GVIP), GVIP Special Issue on Denoising, 2007, pp.1 - 8. www.icgst.com

[3] M. I. H. Bhuiyan, M. O. Ahmad, and M. N. S. Swamy, "A New Homomorphic Bayesian Wavelet-Based MMAE Filter for Despeckling SAR Images”, IEEE International Symposium on Circuits and Systems (ISCAS 2005), 23-26 May 2005, Vol. 5, pp. 4935 - 4938.

[4] M. Do, M. Vetterli, "The contourlet transform: an efficient directional multiresolution image representation", IEEE Transactions on Image Processing, Vol.14, No.12, Dec. 2005, pp. $2091-2106$.

[5] J. J. Ranjani and S. J. Thiruvengadam, "Dual-Tree Complex Wavelet Transform Based SAR Despeckling Using Interscale Dependence", IEEE Transactions on geoscience and remote sensing, Vol. 48, No. 6, June 2010, pp. 2723 - 273.

[6] Z. De-Xiang, W. Xiao, G. Qing, and G. Xiao, "Improving PWF Method of Despeckle in Polarimetric SAR Image by Fusion Based on Nonsubsampled Contourlet Transform", IEEE International Workshop on Education Technology and Training \& International Workshop on Geoscience and Remote Sensing (ETT and GRS 2008), 21-22 Dec. 2008, pp. 328 - 331. 
[7] C. Ding, C. Qu, and F. Su, "Improved Denoising Method of SAR Image", IEEE 3rd International Congress on Image and Signal Processing (CISP 2010), 16-18 Oct. 2010, Vol. 2 , pp. $712-715$.

[8] Z. De-Xiang, GAO Qing-Wei, and WU Xiao-Pei, "SAR Image Despeckling via Bayesian Shrinkage Based on Nonsubsampled Contourlet Transform", IEEE International Conference on Computational and Information Sciences (ICCIS 2010), 17-19 Dec. 2010, pp. 885 - 888.

[9] L. Shahi, H. Behnam, A. Shalbaf, and Z. Alizadeh, "Noise Reduction In Echocardigraphy Images Using Contourlet Transform", IEEE 1st Middle East Conference on Biomedical Engineering (MECBME 2011), 21-24 Feb. 2011,pp. 420 - 423.

[10] P.S. Hiremath, Prema T. Akkasaligar, and S. Badiger, "Performance Comparison of Wavelet Transform and Contourlet Transform based methods for Despeckling Medical Ultrasound Images", International Journal of Computer Applications, Vol. 26, No.9, July 2011, pp. $34-41$.

[11] A. A. Dawood, "A Contourlet-Based Image Denoising Technique with Coefficient Threshold Level Estimation", Tikrit Journal of Engineering Sciences, Vol. 20, No. 4, March 2013, pp. $11-22$.

[12] M. Bhuiyan, M. Ahmad, and M. Swamy, "New Spatially Adaptive Wavelet-based Method for the Despeckling of Medical Ultrasound Images", IEEE International Symposium on Circuits and Systems (ISCAS 2007), 27-30 May 2007, pp. 2347 - 2350.

[13] E. Hui-On Ng," Speckle noise reduction via homomorphic elliptical threshold rotations in the complex wavelet domain", M.Sc. thesis, Electrical and Computer Engineering in the University of Waterloo, Waterloo, Ontario, Canada, 2005.

[14] M. Mastriani," New Wavelet-Based Superresolution Algorithm for Speckle Reduction in SAR Images", International Journal of Computer Science, Vol.1 No. 4, 2007, pp. 291 298.

[15] J. M. Abdul-Jabbar, "Wavelet-Based Homomorphic De-Speckling Technique with Directional Weighted-Averaging Stage”, Al-Rafidain Engineering Journal, Vol. 17, No. 5, Oct. 2009 , pp. $32-43$.

[16] S. Ibrahim, M. Wirth, "Visible and IR Data Fusion Technique Using the Contourlet Transform", IEEE International Conference on Computational Science and Engineering (CSE '09), 29-31 Aug. 2009, Vol. 2, pp. 42 - 47.

[17] S. Wang, X. Xu, B. Hou, and L. Cheng Jiao, "SAR Image Despeckling Using Local Contextual Hidden Markov Model in the Contourlet Domain", CIE International Conference on Radar (ICR '06), 16-19 Oct. 2006, pp. 1 - 4.

[18] A. Mosleh, F. Zargari, R. Azizi, “Texture Image Retrieval Using Contourlet Transform”, IEEE International Symposium on Signals, Circuits and Systems (ISSCS 2009), 9-10 July 2009, pp. $1-4$.

[19] G. Chen, X. Liu, "Contourlet-based Despeckling for SAR Image Using Hidden Markov Tree and Gaussian Markov Models", IEEE 1st Asian and Pacific Conference on Synthetic Aperture Radar (APSAR 2007), 5-9 Nov. 2007, pp. 784 - 787. [20] H. V. Nejad, H. R. Pourreza, and H. Ebrahimi," A novel fuzzy technique for image noise reduction," Proc. of World Academy of Science, Engineering and Technology, Vol.14, August 2006, pp. 390-395.

[21] L. Gagnon and A. Jouan," Speckle filtering of SAR images - A comparative study between complex-wavelet-based and standard filters" Proc. of SPIE, Vol. 3169, pp. 80-91, 1997.

The work was carried out at the college of Engineering. University of Mosul 